\title{
Stochastic Oscillatory Dynamics of Generalized Repressilators
}

\author{
Natalja Strelkowa* and Mauricio Barahona ${ }^{\dagger}$ \\ *Department of Bioengineering, Imperial College London, London, UK \\ ${ }^{\dagger}$ Department of Mathematics, Imperial College London, London, UK
}

\begin{abstract}
We explore the impact of low copy number noise on the onset and quality of oscillations in the generalized repressilator model with odd-number of elements. In our previous work [Strelkowa \& Barahona, 2011] we applied deterministic complexity analysis and provided analytical conditions for the emergence of stable limit cycles via Hopf Bifurcations in oddnumbered rings. Here, we extend this analysis to the stochastic description of the model and study the influence of a biochemical design 'knob' - the gene copy number - on the onset and quality of the oscillations. The gene copy number simultaneously affects two parameters that are usually considered independently in mathematical analyses of this model: namely, the system size $\Omega$ and the deterministic bifurcation parameter $c_{1}$. Here we study the dynamic properties on the ( $\left.\Omega, c_{1}\right)$-plane and characterize how the oscillation characteristics depend on both parameters. The $\left(\Omega, c_{1}\right)$-plane can thus provide a useful perspective for the design and control of engineered synthetic oscillators with respect to biologically meaningful design parameters.
\end{abstract}

Keywords: stochastic Hopf bifurcation, generalized repressilator model, synthetic biology

PACS: $87.10 . \mathrm{Mn}$, 87.10.Tf, 87.85.ff, 87.85.Xd

\section{INTRODUCTION}

Low copy number noise can strongly influence the dynamical behavior of naturally occurring and engineered gene expression networks [1,2]. The origin of noise in genetically identical but phenotypically diverse cells is often attributed to the inherent stochastic nature of biochemical reactions [3, 4].

Synthetically engineered genetic networks can be strongly affected by stochastic fluctuations if they operate in the low copy number regime. Several systems are known in which stochastic fluctuations excite oscillating behavior in genetic circuits $[5,6,7]$ by either effectively stabilizing unstable periodic orbits $[7,6]$ or leading to coherence resonance in the monostable system [5].

The generalized repressilator model, a genetic circuit of coupled repressing genes arranged in the form of a directed ring, has been extensively explored in the deterministic setting using nonlinear complexity analysis $[8,9,7,10]$ and the special case of three genes has already been implemented in living bacterial cells using synthetic biology tools [11]. The model shows qualitatively different steady state characteristics if the ring consists of even number of repressors compared to rings with odd number of repressors [8]. Odd rings are oscillators in which stable oscillations emerge via Hopf bifurcations. The steady state of even rings is a dimerized up/down solution (i.e., they are switches), although even rings are also characterized by the presence of long-lived oscillatory transients that can dominate the observable dynamics in such systems.

In this study, we systematically explore the influence of internal noise on the dynamics of odd rings. In experiments with real bacterial populations, a natural biochemical 'knob' that can be manipulated for parameter and noise control is the gene copy number. However, this biochemical variable simultaneously affects two dynamical parameters in our generalized repressilator model: the system size $\Omega$ and the deterministic bifurcation parameter $c_{1}$, which are usually studied independently in the analysis of these systems. Therefore the dynamical properties of the stochastic generalized repressilator model need to be studied in the $\left(\Omega, c_{1}\right)$-plane.

The linear noise approximation [12] of the stochastic version of the odd-numbered generalized repressilator reveals an interesting phenomenon: the emergence of coherent macroscopic oscillations for parameter values before the deterministic Hopf bifurcation. This effect has been identified previously in other systems undergoing Hopf bifurcations and is attributed to collective microscopic fluctuations which lead to coherent oscillations at the macroscopic level $[13,14,15]$. Since the manipulation of the gene copy number will change the bifurcation parameter in our model, while at the same time changing the level of intrinsic fluctuations present, this leads to an interplay of both effects on the onset of limit cycle oscillations, which can be characterized on the $\left(\Omega, c_{1}\right)$-plane. In particular, we explore how 
internal noise influences the quality of the oscillations through an oscillation quality score in the $\left(\Omega, c_{1}\right)$-plane. Pinpointing such dependencies sheds light on the required parameter manipulation for engineering purposes of synthetic oscillators.

\section{THE GENERALIZED REPRESSILATOR MODEL IN THE MASTER EQUATION FORMALISM}

A simplified stochastic model for the generalized repressilator containing a Monod-type nonlinear transcription term together with linear translation and degradation terms can be derived using time scale separation arguments analogue to those used for the deterministic equation used in the seminal paper by Elowitz and Leibler [11]. Using the creation (annihilation) operators $\mathbb{E}_{p_{j}}\left(\mathbb{E}_{p_{j}}^{-1}\right) / \mathbb{E}_{m_{j}}\left(\mathbb{E}_{m_{j}}^{-1}\right)$ for creation (annihilation) events of proteins and $m R N A$ s (see [12]), the Master equation for the generalized repressilator is given by:

$$
\partial_{t} P(\mathbf{m}, \mathbf{p} ; t)=\left[\frac{c_{1}}{p_{j-1}^{2}+1}\left(\mathbb{E}_{m_{j}}^{-1}-1\right)+c_{2}\left(\mathbb{E}_{m_{j}}-1\right) m_{j}+c_{3} \cdot m_{j}\left(\mathbb{E}_{p_{j}}^{-1}-1\right)+c_{4}\left(\mathbb{E}_{p_{j}}-1\right) p_{j}\right] P(\mathbf{m}, \mathbf{p} ; t),
$$

where the index $j \in\{1, \ldots, N\}$ denotes each of the genes and we consider circular boundary conditions. Note also that the vectors $\mathbf{m}$ and $\mathbf{p}$ are defined over the naturals.

\section{THE DEPENDENCE OF THE REACTION RATES ON THE SYSTEM SIZE, $\Omega$}

The Master equation for the generalized repressilator model contains three types of reactions: two linear terms for the depletion of $m R N A$ s and the creation of protein, and a nonlinear term for the repressor dependent transcription. The rate constants $c_{j}$ in the linear terms do not change with the size of the system $\Omega$ because, in this case, the biochemical rate is just proportional to the number of species in the system, i.e., if the size is increased, so will be the absolute number of species. Therefore the biochemical rate of linear reactions scales with the size of the system [12]. On the other hand, for the nonlinear Monod-type transcription term scaling arguments and derivations van Kampen [12] for extensive and intrinsic variables result in the following transformation rule [16]:

$$
\frac{c_{1}}{1+p^{2}} \rightarrow \frac{c_{1} \Omega}{1+\left(\frac{p}{\Omega}\right)^{2}}
$$

Figure 1 shows simulations of the stochastic system carried out with the Gillespie algorithm at different values of $\Omega$. As expected, if we increase the size of the system $\Omega$, the fluctuations disappear and the oscillations become more regular.
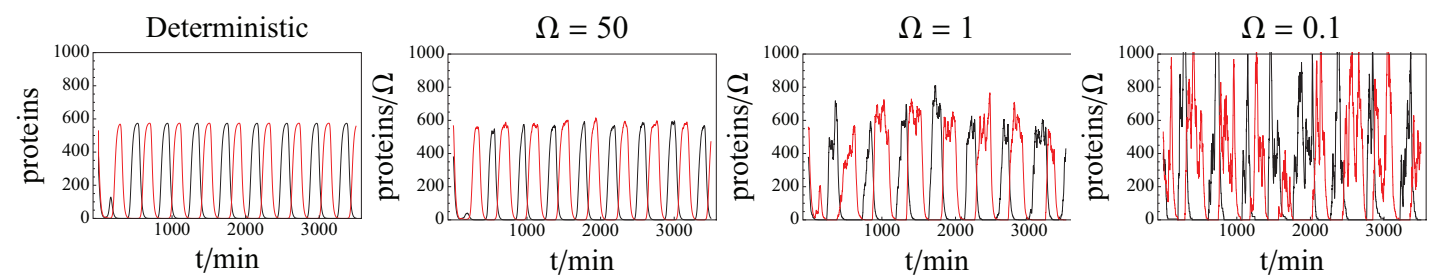

FIGURE 1. From deterministic to stochastic regime in the generalized repressilator model using the dependence of reaction rates on the system size $\Omega$.

The oscillations and their quality are simultaneously affected by changes in the parameter set $\left(c_{i}\right)$ and the system size $\Omega$. A reasonable experimental strategy to sweep the bifurcation parameter $c_{1}$ studied in our earlier work [10] is to change the gene copy number, $d_{0}$, since they are linearly related. The gene copy number $d_{0}$ can be manipulated experimentally and used as a "biological knob" to induce oscillations in synthetic repressilators implemented in living bacterial populations. However, changing $d_{0}$ also affects the system size $\Omega$ [16]. Therefore, the onset of stochastic oscillations in the generalized repressilator model needs to be explored in relation to these two parameters, e. g. in the $\left(\Omega, c_{1}\right)$-plane. 


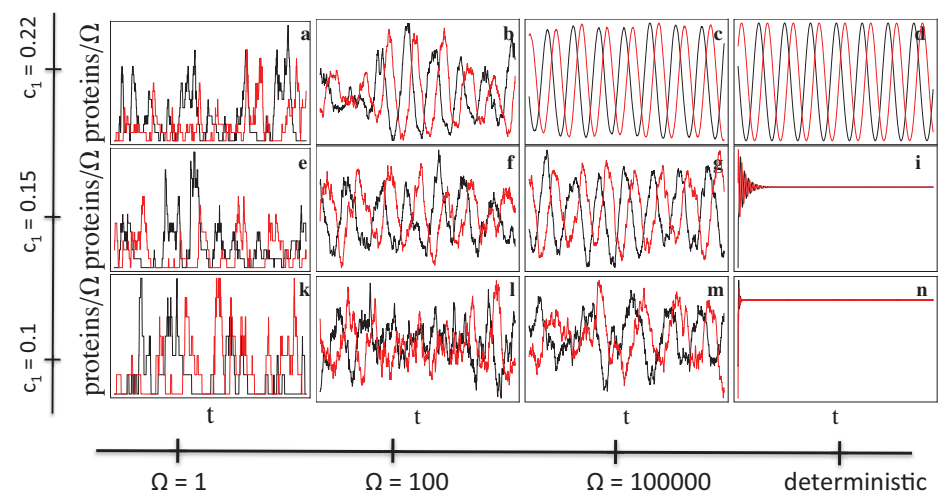

FIGURE 2. Onset of oscillations in the stochastic repressilator model for $N=3$ nearby the deterministic bifurcation point in the $\left(\Omega, c_{1}\right)$ plane. In the deterministic regime, an attracting limit cycle exists for bifurcation parameter $c_{1}>0.21$ leading to periodic behavior. For $c_{1}<0.21$, the system approaches a fixed point. Sub-figures a-d show the behavior of the system after the deterministic Hopf bifurcation and sub-figures e-n before the bifurcation. Depending on the system size $\Omega$, oscillations appear earlier in the stochastic version of the generalized repressilator model $(\mathbf{f}, \mathbf{g})$.

\section{ONSET OF OSCILLATIONS IN THE STOCHASTIC SETTING OF THE GENERALIZED REPRESSILATOR MODEL}

Figure 2 illustrates in the $\left(\Omega, c_{1}\right)$-plane around the onset of oscillations in the stochastic generalized repressilator model. The classical Gillespie algorithm [17] was used for numerical simulations. In the low copy number regime (small $\Omega$ ), where the stochastic fluctuations are strong, the oscillating patterns are less distinct even after the deterministic bifurcation point (Fig. 2a).

An interesting effect occurs in the large- $\Omega$ regime right before the onset of oscillations in the deterministic model (Fig. 2(f,g)). Due to the stochastic broadening of the Hopf bifurcation, oscillations already appear at parameter values below the bifurcation point. This phenomenon has been viewed as an indication that microscopic fluctuations lead to the emergence of coherent oscillations at the macroscopic level around the deterministic bifurcation point in the model [15].

\section{LINEAR NOISE APPROXIMATION CLOSE TO THE HOPF BIFURCATION}

Following the numerical observations in the previous section, we investigate the premature onset of oscillations below the deterministic bifurcation point. We apply the linear noise approximation to the fixed point close to the emergence of the limit cycle close to the Hopf bifurcation (see [12], Chap. X.4).

Denoting the system state $\mathbf{x} \equiv(\mathbf{m}, \mathbf{p})$, the corresponding SDE is

$$
\mathbf{x}(t)=\mathbf{x}_{0}+\int_{0}^{t} J \cdot \mathbf{x} d s+\int_{0}^{t} \sigma \cdot \mathbf{x} d W_{s}
$$

where $J$ is the drift matrix equal to the deterministic Jacobian (see [10]) and $\sigma$ is the diffusion matrix. The stationary stochastic power spectrum of this linear SDE is calculated with the well-known formula for multi-variable linear SDEs ([18], Chap. 4.4.6):

$$
S(v)=(J+i v)^{-1} \sigma \cdot \sigma^{T}(J-i v)^{-1}
$$

The power spectra are obtained from the diagonal contributions on $S(v)$ and shown in Fig. 3 for the fixed point close to the bifurcation point. The power spectrum peak diverges around the resonance values. The linear noise approximation suggests that in all odd-numbered rings, microscopic fluctuations lead to coherent oscillations at the macroscopic level close to the Hopf bifurcation point. Figure 3 illustrates this effect for the ring with $N=3$. 


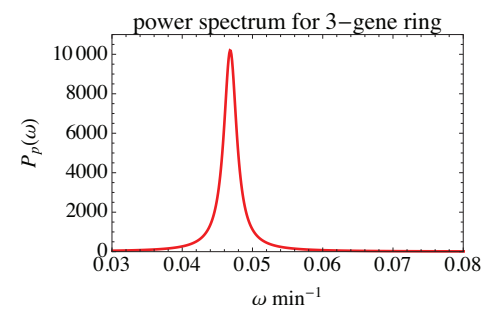

FIGURE 3. Power spectra obtained from the linearization around the fixed point close to the Hopf bifurcation in the generalized repressilator model. In the stochastic system, the oscillations emerge already for parameters below the deterministic Hopf bifurcation. The deterministic bifurcation for $N=3$ occurs at $c_{1}=0.21$. According to the linearized model, we expect coherent stochastic oscillations to appear already at $c_{1}=0.162$ for the stable limit cycle in $N=3$. The frequency peak can be made divergent around this parameter value, which physically means that stochastic fluctuations occurring on the microscopic level lead to coherent oscillations at the macroscopic level [13,14,15]. Direct numerical Gillespie simulations of the system around these values for $N=3$ show oscillations thus confirming the predictions from the linearized spectra.

\section{THE QUALITY OF STOCHASTIC OSCILLATIONS IN THE $\left(\Omega, c_{1}\right)$-PLANE.}

In previous sections we saw that the regularity (or the quality) of stochastic oscillations in the stochastic repressilator model is affected by the system size $\Omega$ and the bifurcation parameter $c_{1}$. To engineer synthetic oscillators in living bacterial cells, it is important to quantitatively explore the quality of oscillations depending on both parameters as they will both be changed through the manipulation of the gene copy number. Therefore, we explore the $\left(\Omega, c_{1}\right)$ plane for the case $N=3$ applying the following quantitative measure [19] for the quality of the oscillations:

$$
Q\left(v_{\text {peak }}\right)=\frac{v_{\text {peak }} S\left(v_{\text {peak }}\right)}{\int S(v) d v}
$$

In the non-oscillating phase $\left(c_{1}=0.1\right)$ the power spectrum is flat, and the quality score assumes its lowest values (Fig. 4). The resonance oscillations prior the Hopf bifurcation point are more prominent in larger systems and a steady increase of the quality score is observed in the second row of the table in Fig. 4 for $c_{1}=0.162$. In the regime long after the bifurcation $\left(c_{1}=1.6\right)$, the quality of oscillations according to this score strongly depends on the system size $\Omega$. In particular, in small systems the quality of oscillations according to this score does not become better even if moving far away from the bifurcation point.

\begin{tabular}{|l|c|c|c|}
\hline & $\Omega=1$ & $\Omega=100$ & $\Omega=100.000$ \\
\hline \hline$c_{1}=1.6$ & $2.4 \cdot 10^{-3}$ & $8.0 \cdot 10^{-2}$ & $1.3 \cdot 10^{-1}$ \\
\hline$c_{1}=0.162$ & $1.0 \cdot 10^{-3}$ & $2.0 \cdot 10^{-3}$ & $3.0 \cdot 10^{-3}$ \\
\hline$c_{1}=0.1$ & $1.4 \cdot 10^{-4}$ & $1.4 \cdot 10^{-3}$ & $1.3 \cdot 10^{-3}$ \\
\hline
\end{tabular}

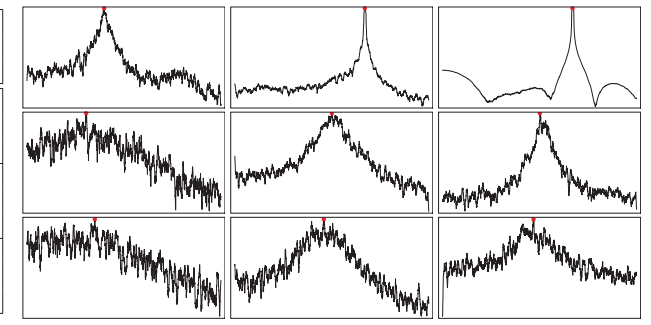

FIGURE 4. Oscillation quality on the $\left(\Omega, c_{1}\right)$-plane. The table shows the quality score defined in Eq. (3) calculated from power spectra obtained from Gillespie simulations of the repressilator model with $N=3$ genes for different system sizes $\Omega \in$ $\{1,100,100.000\}$ with parameters in the non-oscillating phase $\left(c_{1}=0.1\right)$; parameters just prior to the Hopf bifurcation point $\left(c_{1}=0.162\right)$; and parameters clearly above the bifurcation point $\left(c_{1}=1.6\right)$. The (normalized) power spectra obtained from Gillespie simulations, shown on a logarithmic scale, provide an intuition for the oscillation quality score shown in the table.

\section{DISCUSSION}

We have explored the influence of internal noise on the dynamics of the generalized repressilator model. Systematic investigation of the noise influence required the navigation on the $\left(\Omega, c_{1}\right)$-plane, where $\Omega$ is the system size and $c_{1}$ the 
deterministic bifurcation parameter, since they are both dependent on the biologically available gene copy number, $d_{0}$. Both parameters influence the onset as well as the quality of the repressilator oscillations.

For the implementation in living bacterial cells, the gene copy number $d_{0}$ can be seen as a biochemical "knob", which is controlled by the experimentalist and triggers the emergence of Hopf bifurcations in synthetic oscillators inside bacterial cells. In the associated deterministic model, an increase in the gene copy number would in principle just reproduce the sweep of the bifurcation parameter $c_{1}$ shown in the bifurcation diagrams in [7]. However, in the stochastic setting the increase of gene copy numbers has an effect on two model parameters: the bifurcation parameter $c_{1}$ (direct proportionality) as well as the system size $\Omega$ and the associated noise level. The biochemical knob $d_{0}$ can thus be seen as a diagonal navigator on the $\left(\Omega, c_{1}\right)$-plane.

An interesting phenomenon is observed in the stochastic repressilator model just before the onset of the oscillations, e.g. prior the Hopf bifurcation. Coherent oscillations appear in large $\Omega$ regimes already before the deterministic Hopf bifurcation, i.e. at smaller $c_{1}$ values. This effect has been documented in other stochastic systems also right before the deterministic Hopf bifurcation $[13,14,15]$. Intuitively, this can be seen as a driven oscillator operating at the resonance frequency, where the external driving force are the microscopic fluctuations. It is not clear yet if this regime will be observed in real bacterial populations containing such synthetic repressilators and if resonant fluctuations would affect the cells.

Furthermore we investigated the quality of the oscillations in the $\left(\Omega, c_{1}\right)$-plane using a quantitative measure. As expected, in the large $\Omega$-regime the oscillations are of better quality, e.g. more regular. For small system sizes, the quality of the oscillations remains poor even for the parameter sets well beyond the bifurcation point. This effect again emphasizes the importance of the system size as an additional parameter which needs to be considered in synthetic biology designs.

Synthetic repressilators in living bacterial cells should be implemented in sufficiently large $\Omega$ regimes in order to achieve regular oscillations. For the repressilator systems there will always be a trade off between the quality of the oscillations and the amount of metabolic burden due to high levels of expressed heterologous proteins. The $\left(\Omega, c_{1}\right)$ -

plane approach can be used for search of operation regimes with sufficient oscillation quality while achieving the lowest possible metabolic burden.

\section{REFERENCES}

1. A. Raj, and A. van Oudenaarden, Cell 135, 216-226 (2008).

2. A. Eldar, and M. B. Elowitz, Nature 467, 167-173 (2010).

3. J. M. Raser, and E. K. O'Shea, Science 309, 2010-2013 (2005).

4. T. J. Perkins, and P. S. Swain, Mol Syst Biol 5, 326 (2009).

5. H. El-Samad, and M. Khammash, Proc. of the 45th IEEE Conf. on Decision and Control (2006).

6. M. Turcotte, J. Garcia-Ojalvo, and G. M. Süel., Proc. Natl. Acad. Sci. USA 105, 15732-15737 (2008).

7. N. Strelkowa, and M. Barahona, Journal of The Royal Society Interface 7, 1071-1082 (2010).

8. H. Smith, J. Math. Biol. 25, 169-190 (1987).

9. S. Müller, J. Hofbauer, L. Endler, C. Flamm, S. Widder, and P. Schuster, J. Math. Biol. 53, 905-937 (2006).

10. N. Strelkowa, and M. Barahona, Chaos: An Interdisciplinary Journal of Nonlinear Science 21, 023104 (2011).

11. M. B. Elowitz, and S. Leibler, Nature 403, 335-338 (2000).

12. N. G. van Kampen, Stochastic Processes in Physics and Chemistry., Elsevier, Amsterdam, 2007, 3-rd edition edn.

13. A. J. McKane, and T. J. Newman, Phys Rev Lett 94, 218102 (2005).

14. R. Kuske, L. F. Gordillo, and P. Greenwood, Journal of Theoretical Biology 245, 459 - 469 (2007).

15. R. P. Boland, T. Galla, and A. J. McKane, J. of Statistical Mechanics: Theory and Experiment 2008, P09001 (2008).

16. N. Strelkowa, Stochastic analysis of nonlinear dynamics and feedback control for gene regulatory networks with applications to synthetic biology, Ph.D. thesis, Imperial College London (2011).

17. D. T. Gillespie, J. Chem. Phys. 8, 2340-2361 (1977).

18. C. W. Gardiner, Handbook of Stochastic Methods, Springer, 2004, 3-rd edn.

19. S. Risau-Gusman, and G. Abramson, The European Physical Journal B 60, 515-520 (2007). 